\title{
In Silico Characterization of miRNA and Long Non-Coding RNA Interplay in Multiple Myeloma
}

\author{
Domenica Ronchetti ${ }^{1,2}$, Martina Manzoni ${ }^{1,2}$, Katia Todoerti ${ }^{3}$, Antonino Neri ${ }^{1,2, *}$ and \\ Luca Agnelli ${ }^{1,2}$,* \\ 1 Department of Oncology and Hemato-Oncology, University of Milano, 20122 Milan, Italy; \\ domenica.ronchetti@unimi.it (D.R.); martina.manzoni@unimi.it (M.M.) \\ 2 Hematology Unit, Fondazione IRCCS Ca' Granda, Ospedale Maggiore Policlinico, 20122 Milan, Italy \\ 3 Laboratory of Pre-Clinical and Translational Research, IRCCS-CROB, Referral Cancer Center of Basilicata, \\ 85028 Rionero in Vulture (PZ), Italy; katia.todoerti@gmail.com \\ * Correspondence: antonino.neri@unimi.it (A.N.); luca.agnelli@gmail.com (L.A.); \\ Tel.: +39-02-5032-0420 (A.N.); +39-02-5503-3328 (L.A.)
}

Academic Editor: George A. Calin

Received: 11 October 2016; Accepted: 21 November 2016; Published: 29 November 2016

\begin{abstract}
The identification of deregulated microRNAs (miRNAs) and long non-coding RNAs (lncRNAs) in multiple myeloma (MM) has progressively added a further level of complexity to MM biology. In addition, the cross-regulation between lncRNAs and miRNAs has begun to emerge, and theoretical and experimental studies have demonstrated the competing endogenous RNA (ceRNA) activity of lncRNAs as natural miRNA decoys in pathophysiological conditions, including cancer. Currently, information concerning lncRNA and miRNA interplay in MM is virtually absent. Herein, we investigated in silico the lncRNA and miRNA relationship in a representative datasets encompassing $95 \mathrm{MM}$ and 30 plasma cell leukemia patients at diagnosis and in four normal controls, whose expression profiles were generated by a custom annotation pipeline to detect specific lncRNAs. We applied target prediction analysis based on miRanda and RNA22 algorithms to 235 lncRNAs and 459 miRNAs selected with a potential pivotal role in the pathology of MM. Among pairs that showed a significant correlation between lncRNA and miRNA expression levels, we identified 11 lncRNA-miRNA relationships suggestive of a novel ceRNA network with relevance in MM.
\end{abstract}

Keywords: multiple myeloma; lncRNA; miRNA

\section{Introduction}

Multiple myeloma (MM) is a malignant proliferation of bone marrow (BM) plasma cells (PCs) characterized by a highly heterogeneous genetic background and clinical course, ranging from pre-malignant monoclonal gammopathy of undetermined significance to smoldering MM, symptomatic MM, and plasma cell leukemia (PCL). Approximately half of MM tumors present a hyperdiploid karyotype generally in association with a better prognosis, whereas non-hyperdiploid tumors are enriched in primary IGH translocations events [1]. The identification of overexpressed microRNAs (miRNAs) in MM has undoubtedly contributed to MM molecular classification. Specific miRNAs have been identified as deregulated in distinct subgroups of MM patients, mainly in association with IGH translocations or allelic imbalances, and individual miRNAs have been demonstrated to be involved in neoplastic transformation and the progression of the disease [2-7].

miRNAs can regulate gene expression at the post-transcriptional level through binding to miRNA response elements (MREs) in the untranslated regions or the coding sequence of target genes [8]. It has been shown that different RNA molecules harboring MREs can compete for a common pool of miRNAs, thus acting as competing endogenous RNAs (ceRNAs) $[9,10]$. There is increasing evidence that ceRNA 
crosstalk occurs widely in cellular processes, and its perturbation will unsettle the transcriptomic equilibrium leading to disease initiation and progression $[10,11]$. The miRNA-mediated circuits among transcripts can involve coding as well as noncoding transcripts, including long noncoding RNAs (lncRNAs), which are ncRNAs larger than 200 nucleotides in length [12-14]. IncRNAs act as essential components of complex gene regulatory network by regulating gene expression at the transcriptional, post-transcriptional, and epigenetic levels $[15,16]$. Thousands of lncRNAs have been annotated in eukaryotic genomes (annotation from LncRNAdb, Ensembl, Broad Institute, Gencode 13, Refseq, and NONCODE collected in LNCipedia repository [17]), many of which are preferentially located in the cytoplasm [18], where they could be engaged in miRNA-mediated interactions with other transcripts. Both computational and experimental evidence support the extensive targeting of lncRNAs by miRNAs [19]. Recent studies have demonstrated the ceRNA activity of lncRNAs as natural miRNA decoys in human development and pathophysiological conditions [20]. Systematic analyses of IncRNA-associated ceRNA network have been performed in breast cancer [21,22], gastric cancer [23], and glioblastoma multiforme [24]. Recently, we and others have described the pattern of lncRNAs deregulation in MM distinct subgroups [25] and in human MM cell lines resistant to proteasome inhibitors [26]; however, a portrait of lncRNA and miRNA interplay in the pathology is still lacking.

The present study was therefore aimed at investigating in silico the lncRNA and miRNA relationship in a cohort of MM patients and in normal BM PCs. We searched for putative significant correlation between lncRNAs and miRNAs expression level in paired samples, with the support of target prediction analysis. Overall, our findings identified lncRNA-miRNA pairs suggestive of a novel ceRNA network with a potential impact in MM biology.

\section{Materials and Methods}

\subsection{Patients}

The study included bone marrow aspirates from newly diagnosed $95 \mathrm{MM}$ and 30 PCL patients obtained during standard diagnostic procedures at the IRCCS Institution in Milan, as previously reported [3]. Patients employed for the study were representative of the major molecular characteristics of the disease. Samples were characterized for the presence of the most frequent chromosomal translocations and the ploidy status based on fluorescence in situ hybridization (FISH) evaluation criteria, as previously described [27]. Written informed consent was obtained from all patients in accordance with the declaration of Helsinki. Four normal controls were purchased from Voden, Medical Instruments IT.

\subsection{Expression Profiling}

miRNA and lncRNA expression data was available for 95MM, 30 PCL, and 4 normal samples (GSE87830). Normal sample RNAs were collected from four normal bone marrow donors (Voden, Medical Instruments IT, Meda, Milan, Italy). miRNA profiling was generated using GeneChip ${ }^{\circledR}$ miRNA 3.0 Array (Affymetrix Inc., Santa Clara, CA, USA) as previously described [3]. IncRNAs expression data of the paired samples were extracted from CEL files generated on GeneChip ${ }^{\circledR}$ Gene 1.0 ST Array (Affymetrix Inc., Santa Clara, CA, USA) as previously reported [28]. Briefly, expression data have been normalized using an RMA procedure at the probe cluster ID annotation level. Cross-hybridization probes were filtered out. We combined annotated probes with both Ensembl transcripts (GRCh37/hg19 assembly, http:/ /grch37.ensembl.org/index.html) and lncRNAs from the version 4.0 of the LNCipedia repository database (http://www.lncipedia.org/), based on the chromosome localization of the target sequence identified by each probe; afterwards, we considered only probes univocally referable to lncRNA transcripts (i.e., that do not overlap with Ensembl transcripts). To summarize probes related to each lncRNA, we considered their median expression value [25]. The differentially expressed miRNAs and lncRNAs discriminating MM PCs and normal PC counterparts were identified with Significant 
Analysis of Microarrays (SAM) software version 5.00 [29] using the web application provided in the shiny package for R software [30].

\subsection{Statistical Analysis}

A Wilcoxon rank-sum test was applied using standard functions in the $\mathrm{R}$ base package. The Benjamini-Hochberg method was applied for multiple testing correction. miRNA targets custom predictions were obtained for lncRNA sequences by merging the results of two different algorithms: the RNA-22 version 2.0 prediction algorithm [31] and miRanda [32], which both allow customizing input sequences and parameters. The RNA-22 perl script was run on the combination of the 459 miRNA and 1546 (number of different transcripts corresponding to 235 lncRNAs) lncRNA FASTA sequences selected as relevant among all miRNAs and lncRNAs respectively detected by the arrays. Default sensitivity/specificity ratio of 1.032 was chosen, provided a minimum seed size of 7 bases with only one mismatch exception, a minimum number of 12 paired-up bases in heteroduplex with $-12 \mathrm{Kcal} / \mathrm{mol}$ maximum folding energy, and no more than one G-U wobble in the seed region. The miRanda algorithm was run under default conditions, without any a priori restrictions on score, energy, or trimming parameters.

\section{Results}

\subsection{IncRNA and miRNA Interplay in $M M$}

\subsubsection{Selection of Relevant lncRNAs and miRNAs}

The expression profiles of lncRNAs and miRNAs were investigated in a cohort of $95 \mathrm{MM}$ and four normal controls. To be confident in the detection of specific lncRNAs, we applied a custom annotation pipeline that remapped the probes included in the original array to distinct lncRNAs, according to the last updated LNCipedia-v4 database genomic annotations. Such a strategy led us to investigate the expression levels of 1614 well-annotated and specific human lncRNAs. Concerning miRNAs, the arrays could detect 1768 mature miRNAs. To focus on relevant lncRNA-miRNA interactions, the full lists of detectable transcripts were subsequently shortened according to the following criteria. First, we included in each list the lncRNAs and miRNAs suggestive of a role in the pathogenesis of MM by selecting those lncRNAs and miRNAs that resulted as differentially expressed in a supervised comparison between MM and normal PCs (setting the threshold for the 90th percentile of false discovery rate to the null value). Next, based on the highly heterogeneous genetic background of MM, all lncRNAs and miRNAs fulfilling a variational filter were included. From the resulting list of lncRNAs, we excluded those located in chromosomal regions, such as $14 q 32,2 p$, and 22q, coding for the highly variable portions of the immunoglobulin genes or for IGHV pseudogenes (named Ab-parts), whose expression is related to the highly complex transcriptional activity of such regions to achieve a polyclonal antibody repertoire. Ultimately, for further analyses, we focused on 235 lncRNAs and 459 miRNAs (Figure 1, Supplementary Materials Table S1).

\subsubsection{Identification of lncRNAs miRNA-Target}

To identify the relationship between lncRNAs and miRNAs, we first investigated which IncRNAs could be a potential miRNA target. Two of the most common target prediction algorithms (RNA-22 and miRanda) were run on each miRNA/lncRNA from the above-defined lists, testing the miRNA sequences annotated on miRbase v20 against the LNCipedia-annotated lncRNA sequences corresponding to the fragments investigated by the probes on the array. Hence, the target prediction analysis is able to specify which of the different transcripts, if any, of each of the 235 lncRNA was targeted by any of the 459 miRNAs. We identified 12,844 lncRNA-miRNA pairs supported by both target prediction algorithms (after correction of RNA22-derived data at FDR $<5 \%$ for multiple testing). 
Specifically, the obtained pairs represented the combination of 410 miRNAs and different transcripts from 113 lncRNAs.

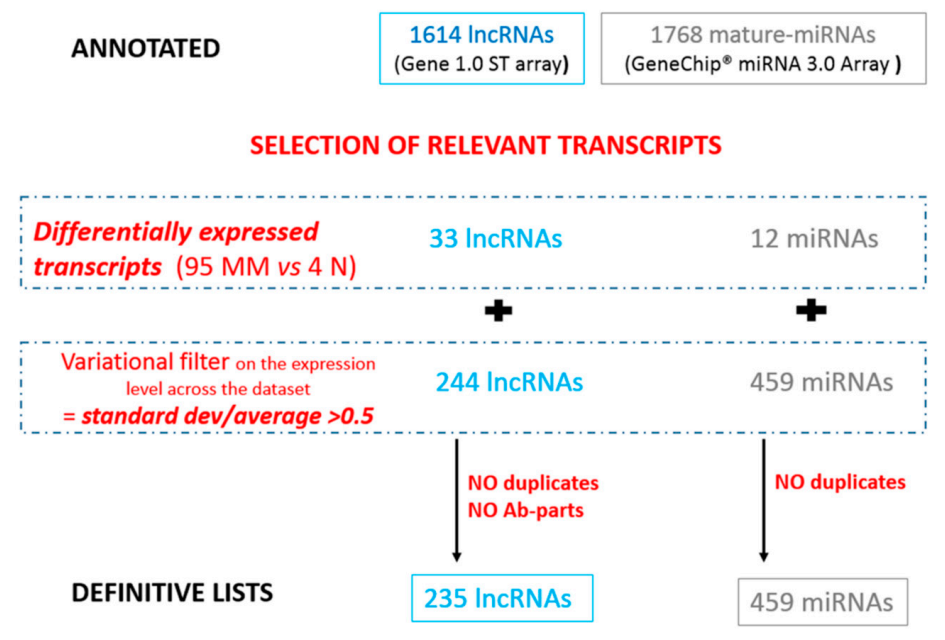

Figure 1. Flow chart used to select relevant lncRNAs and miRNAs for the following analyses.

\subsubsection{Correlation between lncRNAs and Corresponding miRNA Targets in MM}

Therefore, to test the occurrence of a potential transcriptional association between lncRNAs and miRNAs, we investigated the correlations between the expression levels of each miRNA/lncRNA defined above in all the patients of the cohort. We identified 11 lncRNA-miRNA pairs for which the target relationship has been predicted and whose expression was significantly anti-correlated in our database $(q$-value $<0.05)$ (Table 1$)$.

Table 1. Target prediction metrics of correlated lncRNA-miRNAs pairs.

\begin{tabular}{|c|c|c|c|c|c|c|c|}
\hline \multirow[b]{2}{*}{ lncRNA } & \multirow[b]{2}{*}{$\begin{array}{l}\text { IncRNA } \\
\text { Chr. }{ }^{1}\end{array}$} & \multirow[b]{2}{*}{ miRNA } & \multirow{2}{*}{$\begin{array}{c}\text { miRNA } \\
\text { Chr. }^{1}\end{array}$} & \multicolumn{2}{|c|}{ Correlation } & \multicolumn{2}{|c|}{ Target Prediction $^{2}$} \\
\hline & & & & $\begin{array}{l}\text { Corr. } \\
\text { Coeff. }\end{array}$ & $q$-val. & $\begin{array}{l}\text { lncRNA Target } \\
\text { Sequence from/to }\end{array}$ & $q$-val. \\
\hline DLEU2:26 & $13 q 14$ & mir-3175 & $15 q 26$ & -0.35 & 0.0444 & $668-690$ & 0.011 \\
\hline KLF3-AS1:2 & $4 \mathrm{p} 14$ & $\operatorname{mir}-4787-5 p$ & $3 \mathrm{p} 21$ & -0.36 & 0.0319 & 1890-1911 & 0.045 \\
\hline LINC00173:6 & $12 \mathrm{q} 24$ & mir-221-3p & Xp11 & -0.49 & 0.0003 & $2485-2506$ & 0.013 \\
\hline LINC00173:9 & $12 \mathrm{q} 24$ & mir-221-3p & Xp11 & -0.49 & 0.0003 & 10737-10759 & 0.019 \\
\hline AGBL1-4 & $15 q 25.3$ & mir-185-5p & $22 q 11$ & -0.37 & 0.0266 & $3303-3321$ & 0.040 \\
\hline MCL1-2:1 & $1 \mathrm{q} 21$ & mir-106a-5p & $\mathrm{Xq} 26$ & -0.36 & 0.0351 & $5285-5306$ & $<1 \mathrm{E}-06$ \\
\hline MCL1-2:1 & $1 \mathrm{q} 21$ & mir-18a-5p & $13 q 31$ & -0.43 & 0.0041 & $4377-4397$ & 0.043 \\
\hline MCL1-2:1 & $1 \mathrm{q} 21$ & mir-18b-5p & $\mathrm{Xq} 26$ & -0.35 & 0.0388 & $2903-2923$ & 0.023 \\
\hline MCL1-2:1 & $1 \mathrm{q} 21$ & mir-20a-5p & $13 q 31$ & -0.36 & 0.0351 & $98-120$ & 0.047 \\
\hline MCL1-2:1 & $1 \mathrm{q} 21$ & $\operatorname{mir}-17-5 p$ & $13 q 31$ & -0.35 & 0.0449 & 184-206 & 0.011 \\
\hline WDR73-3:10 & $15 q 25.2$ & mir-423-5p & 17q11 & -0.40 & 0.0124 & 2090-2113 & 0.049 \\
\hline
\end{tabular}

\subsection{IncRNA and miRNA Interplay in PCL}

To investigate whether the interplay of lncRNAs and miRNAs identified in MM may occur in other forms of plasma cell dyscrasia, we evaluated the correlation between the expression levels of the pairs reported in Table 1 in a cohort of 30 PCL profiled for lncRNA and miRNA expressions as previously described. Notably, lnc-MCL1-2 expression was significantly anti-correlated with those of mir-106a-5p, mir-18a-5p, and mir-18b-5p, and lnc-AGBL1-4 with mir-185-5p (Table 2). 
Table 2. lncRNAs highly correlated with miRNAs in PCL.

\begin{tabular}{cccc}
\hline miRNA & lncRNA Transcript & Corr. Coeff. & $p$-val. \\
\hline hsa-mir-185-5p & lnc-AGBL1-4:1 & -0.451 & 0.012 \\
hsa-mir-106a-5p & lnc-MCL1-2:1 & -0.377 & 0.039 \\
hsa-mir-18a-5p & lnc-MCL1-2:1 & -0.431 & 0.017 \\
hsa-mir-18b-5p & lnc-MCL1-2:1 & -0.466 & 0.009 \\
\hline
\end{tabular}

\section{Discussion}

Like most human cancers, $\mathrm{MM}$ is frequently associated with an altered transcription pattern involving both protein-coding RNAs [33,34], and multiple noncoding members [5,25,35]. Recently, we reported the deregulation of lncRNAs in all major forms of plasma cell dyscrasia as compared to healthy controls; in particular, distinct lncRNA transcriptional fingerprints characterize specific MM subgroups. Furthermore, we found lncRNAs whose expression was progressively deregulated in association with more aggressive stages of the pathology, suggesting a possible role in the progression of the disease [25].

lncRNA and miRNAs have been established as key players in regulating various biological and pathological processes, such as cell-cycle progression, chromatin remodeling, gene transcription, and posttranscriptional processing; furthermore, the cross-regulation between IncRNAs and miRNAs has emerged $[9,10,36]$.

The present study provides an unprecedented overview of the miRNA/lncRNA relationship in multiple myeloma. We have investigated in silico the lncRNA and miRNA potential connection in representative cohorts of MM and PCL patients and in normal PC controls, and identified lncRNA-miRNA pairs that may have impact in the pathology.

One of the most suggestive findings involves lnc-MCL1-2, which was both significantly anti-correlated to and predicted target of miRNAs belonging to mir-17 gene family. lnc-MCL1-2 maps less than $2 \mathrm{~kb}$ centromeric to the Myeloid Cell Leukemia (MCL1) gene: their expression levels highly correlate in our dataset $(R=0.52)$. In addition, members of the mir-17 family such as mir-106a-5p, mir-18a-5p, mir18b-5p, and mir-17-5p have been reported to target MCL1 [37,38]. Based on these data, we can hypothesize a ceRNA mechanism in which lnc-MCL1-2 acts as sponge for mir-17 miRNAs family, thus regulating MCL1 transcription. This putative ceRNA network may play a role in the pathology, as MCL1 has been recently described as a pivotal gene for maintaining cell survival [39]. Likewise, anti-correlated expression levels of lnc-MCL1-2 and members of the mir-17 gene family were also found in PCL, thus suggesting that the importance of this interplay may be extended to other forms of PC dyscrasia.

mir-185-5p is significantly anti-correlated and predicted to target lnc-AGBL1-4, which had been found to be upregulated in MM PCs compared to normal controls [25]. As regards this pair, we established a transcriptional relationship in PCL, too. lnc-AGBL1-4 (also named LINC00052) is located less than $300 \mathrm{~kb}$ centromeric to the Neurotrophic Tyrosine Kinase Receptor Type 3 (NTRK3) gene, already known to be involved in carcinogenesis. In fact, mutated NTRK3 gene and ETV6-NTRK3 gene fusion following $\mathrm{t}(12 ; 15)$ chromosomal translocation are associated with different types of tumors, including leukemia [40,41]. Interestingly, in hepatocellular carcinoma, lnc-AGBL1-4 has been demonstrated to regulate the expression of NTRK3 by miR-128 and miR-485-3p to strengthen cell invasion and migration [42]. In addition, the direct targeting of mir-185-5p to NTRK3 has been demonstrated in neuroblastoma [43]. These data suggest that, in MM and PCL, a circuitry might exist that involves lnc-AGBL1-4 and mir-185-5p ultimately leading to NTRK3 expression regulation. Although the expression levels of lnc_AGBL1-4 and NTRK3 were not significantly correlated in our cohort of patients, all these considerations prompt further verification of this relationship in MM.

Additional studies merit the pairs lnc-DLEU2/mir-3175, lnc-WDR73-3/mir-423-5p, and LINC00173/mir-221-3p, as all these lncRNAs were found significantly deregulated in specific 
molecular MM subgroups in our previous investigations [25]. Specifically, lnc-DLEU2 was upregulated in MM patients with the $t(11 ; 14)$ translocation and downregulated in samples with the deletion of chromosome 13q; lnc-WDR73-3 was downregulated in MM samples with the $\mathrm{t}(11 ; 14)$ translocation; LINC00173 was upregulated in patients overexpressing MAF genes in association with the mir-221 downregulation in the same MM subgroups. This is of particular interest because the cluster miR-221/222 is strongly upregulated in a variety of solid and hematologic malignancies, and mir-221/222 inhibitors exert anti-tumor activity in vitro and in vivo in MM [44]. Understanding the interplay with LINC00173 may unravel novel mechanisms on which the modulation of mir-221 relies and might ultimately provide the rationale to investigate innovative target therapies.

\section{Conclusions}

Herein, we have provided a portrait of the most reliable lncRNA/miRNA relationships in a representative cohort of primary MM tumors by means of in silico analysis based on the integration of expression data with target predictions. Of those identified, conceivably relevant interactions for MM biology were established between lnc-MCL1-2 and mir-17 gene family, lnc-AGBL1-4 and mir-185-5p, lnc-DLEU2 and miR-3175, LINC00173 and miR-221. Overall, our study reinforce the notion that understanding the RNA crosstalk will lead to significant insights into gene regulatory networks and will contribute to the comprehension of MM patho-physiology.

Supplementary Materials: The following are available online at www.mdpi.com/2073-4425/7/12/107/s1. Table S1: List of selected relevant miRNAs and lncRNAs.

Acknowledgments: This work was supported by grants from Associazione Italiana Ricerca sul Cancro (AIRC) to Antonino Neri (IG16722, IG10136, and the "Special Program Molecular Clinical Oncology—5 per mille" n. 9980, 2010/15); Katia Todoerti was supported by the Italian Health Minister, Finalized Research for Young Researchers, CUP Project E66110000230001.

Author Contributions: D.R. and L.A. conceived and designed the experiments, D.R. M.M. K.T., and L.A. analyzed the data; A.N. contributed reagents/materials/analysis tools; D.R., L.A., and A.N. wrote the paper.

Conflicts of Interest: The authors declare no conflict of interest.

\section{References}

1. Morgan, G.J.; Walker, B.A.; Davies, F.E. The genetic architecture of multiple myeloma. Nat. Rev. Cancer 2012, 12, 335-348. [CrossRef] [PubMed]

2. Agnelli, L.; Tassone, P.; Neri, A. Molecular profiling of multiple myeloma: From gene expression analysis to next-generation sequencing. Expert. Opin. Biol. Ther. 2013, 13, S55-S68. [CrossRef] [PubMed]

3. Calura, E.; Bisognin, A.; Manzoni, M.; Todoerti, K.; Taiana, E.; Sales, G.; Morgan, G.J.; Tonon, G.; Amodio, N.; Tassone, P.; et al. Disentangling the microRNA regulatory milieu in multiple myeloma: Integrative genomics analysis outlines mixed miRNA-TF circuits and pathway-derived networks modulated in $\mathrm{t}(4 ; 14)$ patients. Oncotarget 2016, 7, 2367-2378. [PubMed]

4. Gutierrez, N.C.; Sarasquete, M.E.; Misiewicz-Krzeminska, I.; Delgado, M.; De Las, R.J.; Ticona, F.V.; Ferminan, E.; Martin-Jimenez, P.; Chillon, C.; Risueno, A.; et al. Deregulation of microRNA expression in the different genetic subtypes of multiple myeloma and correlation with gene expression profiling. Leukemia 2010, 24, 629-637. [CrossRef] [PubMed]

5. Lionetti, M.; Biasiolo, M.; Agnelli, L.; Todoerti, K.; Mosca, L.; Fabris, S.; Sales, G.; Deliliers, G.L.; Bicciato, S.; Lombardi, L.; et al. Identification of microRNA expression patterns and definition of a microRNA/mRNA regulatory network in distinct molecular groups of multiple myeloma. Blood 2009, 114, e20-e26. [CrossRef] [PubMed]

6. Lionetti, M.; Agnelli, L.; Lombardi, L.; Tassone, P.; Neri, A. MicroRNAs in the pathobiology of multiple myeloma. Curr. Cancer Drug Targets 2012, 12, 823-837. [CrossRef] [PubMed]

7. Zhou, Y.; Chen, L.; Barlogie, B.; Stephens, O.; Wu, X.; Williams, D.R.; Cartron, M.A.; van, R.F.; Nair, B.; Waheed, S.; et al. High-risk myeloma is associated with global elevation of miRNAs and overexpression of EIF2C2/AGO2. Proc. Natl. Acad. Sci. USA 2010, 107, 7904-7909. [CrossRef] [PubMed] 
8. Cai, Y.; Yu, X.; Hu, S.; Yu, J. A brief review on the mechanisms of miRNA regulation. Genom. Proteom. Bioinf. 2009, 7, 147-154. [CrossRef]

9. Salmena, L.; Poliseno, L.; Tay, Y.; Kats, L.; Pandolfi, P.P. A ceRNA hypothesis: the Rosetta Stone of a hidden RNA language? Cell 2011, 146, 353-358. [CrossRef] [PubMed]

10. Tay, Y.; Rinn, J.; Pandolfi, P.P. The multilayered complexity of ceRNA crosstalk and competition. Nature 2014, 505, 344-352. [CrossRef] [PubMed]

11. Karreth, F.A.; Pandolfi, P.P. ceRNA cross-talk in cancer: When ce-bling rivalries go awry. Cancer Discov. 2013, 3, 1113-1121. [CrossRef] [PubMed]

12. Cesana, M.; Cacchiarelli, D.; Legnini, I.; Santini, T.; Sthandier, O.; Chinappi, M.; Tramontano, A.; Bozzoni, I. A long noncoding RNA controls muscle differentiation by functioning as a competing endogenous RNA. Cell 2011, 147, 358-369. [CrossRef] [PubMed]

13. Franco-Zorrilla, J.M.; Valli, A.; Todesco, M.; Mateos, I.; Puga, M.I.; Rubio-Somoza, I.; Leyva, A.; Weigel, D.; Garcia, J.A.; Paz-Ares, J. Target mimicry provides a new mechanism for regulation of microRNA activity. Nat. Genet. 2007, 39, 1033-1037. [CrossRef] [PubMed]

14. Wang, Y.; Xu, Z.; Jiang, J.; Xu, C.; Kang, J.; Xiao, L.; Wu, M.; Xiong, J.; Guo, X.; Liu, H. Endogenous miRNA sponge lincRNA-RoR regulates Oct4, Nanog, and Sox2 in human embryonic stem cell self-renewal. Dev. Cell 2013, 25, 69-80. [CrossRef] [PubMed]

15. Mercer, T.R.; Mattick, J.S. Structure and function of long noncoding RNAs in epigenetic regulation. Nat. Struct. Mol. Biol. 2013, 20, 300-307. [CrossRef] [PubMed]

16. Yoon, J.H.; Abdelmohsen, K.; Gorospe, M. Posttranscriptional gene regulation by long noncoding RNA. J. Mol. Biol. 2013, 425, 3723-3730. [CrossRef] [PubMed]

17. LNCipedia repository. Available online: http://www.lncipedia.org/ (accessed on 1 August 2016).

18. van, H.S.; van, I.M.; Jacobi, J.; Boymans, S.; Essers, P.B.; de, B.E.; Hao, W.; MacInnes, A.W.; Cuppen, E.; Simonis, M. Extensive localization of long noncoding RNAs to the cytosol and mono- and polyribosomal complexes. Genom. Biol. 2014, 15, R6-R17.

19. Paraskevopoulou, M.D.; Georgakilas, G.; Kostoulas, N.; Reczko, M.; Maragkakis, M.; Dalamagas, T.M.; Hatzigeorgiou, A.G. DIANA-LncBase: Experimentally verified and computationally predicted microRNA targets on long non-coding RNAs. Nucleic Acids Res. 2013, 41, D239-D245. [CrossRef] [PubMed]

20. Sanchez-Mejias, A.; Tay, Y. Competing endogenous RNA networks: Tying the essential knots for cancer biology and therapeutics. J. Hematol. Oncol. 2015, 8, 30-38. [CrossRef] [PubMed]

21. Paci, P.; Colombo, T.; Farina, L. Computational analysis identifies a sponge interaction network between long non-coding RNAs and messenger RNAs in human breast cancer. BMC Syst. Biol. 2014, 8, 83-97. [CrossRef] [PubMed]

22. Zhou, X.; Liu, J.; Wang, W. Construction and investigation of breast-cancer-specific ceRNA network based on the mRNA and miRNA expression data. IET Syst. Biol. 2014, 8, 96-103. [CrossRef] [PubMed]

23. Xia, T.; Liao, Q.; Jiang, X.; Shao, Y.; Xiao, B.; Xi, Y.; Guo, J. Long noncoding RNA associated-competing endogenous RNAs in gastric cancer. Sci. Rep. 2014, 4, 6088-6094. [CrossRef] [PubMed]

24. Chiu, Y.C.; Hsiao, T.H.; Chen, Y.; Chuang, E.Y. Parameter optimization for constructing competing endogenous RNA regulatory network in glioblastoma multiforme and other cancers. BMC Genomics 2015, 16, S1-S13. [CrossRef] [PubMed]

25. Ronchetti, D.; Agnelli, L.; Taiana, E.; Galletti, S.; Manzoni, M.; Todoerti, K.; Musto, P.; Strozzi, F.; Neri, A. Distinct lncRNA transcriptional fingerprints characterize progressive stages of multiple myeloma. Oncotarget 2016, 7, 14814-14830. [PubMed]

26. Malek, E.; Kim, B.G.; Driscoll, J.J. Identification of Long Non-Coding RNAs Deregulated in Multiple Myeloma Cells Resistant to Proteasome Inhibitors. Genes 2016, 7, 84. [CrossRef] [PubMed]

27. Agnelli, L.; Fabris, S.; Bicciato, S.; Basso, D.; Baldini, L.; Morabito, F.; Verdelli, D.; Todoerti, K.; Lambertenghi-Deliliers, G.; Lombardi, L.; et al. Upregulation of translational machinery and distinct genetic subgroups characterise hyperdiploidy in multiple myeloma. Br. J. Haematol. 2007, 136, 565-573. [CrossRef] [PubMed]

28. Todoerti, K.; Agnelli, L.; Fabris, S.; Lionetti, M.; Tuana, G.; Mosca, L.; Lombardi, L.; Grieco, V.; Bianchino, G.; D'Auria, F; et al. Transcriptional characterization of a prospective series of primary plasma cell leukemia revealed signatures associated with tumor progression and poorer outcome. Clin. Cancer Res. 2013, 19, 3247-3258. [CrossRef] [PubMed] 
29. Tusher, V.G.; Tibshirani, R.; Chu, G. Significance analysis of microarrays applied to the ionizing radiation response. Proc. Natl. Acad. Sci. USA 2001, 98, 5116-5121. [CrossRef] [PubMed]

30. MikeJSeo/SAM. Available online: https://github.com/MikeJSeo/SAM (accessed on 1 August 2016).

31. Miranda, K.C.; Huynh, T.; Tay, Y.; Ang, Y.S.; Tam, W.L.; Thomson, A.M.; Lim, B.; Rigoutsos, I. A pattern-based method for the identification of MicroRNA binding sites and their corresponding heteroduplexes. Cell 2006, 126, 1203-1217. [CrossRef] [PubMed]

32. Betel, D.; Wilson, M.; Gabow, A.; Marks, D.S.; Sander, C. The microRNA.org resource: Targets and expression. Nucleic Acids Res. 2008, 36, D149-D153. [CrossRef] [PubMed]

33. Agnelli, L.; Bicciato, S.; Mattioli, M.; Fabris, S.; Intini, D.; Verdelli, D.; Baldini, L.; Morabito, F.; Callea, V.; Lombardi, L.; et al. Molecular classification of multiple myeloma: A distinct transcriptional profile characterizes patients expressing CCND1 and negative for 14q32 translocations. J. Clin. Oncol. 2005, 23, 7296-7306. [CrossRef] [PubMed]

34. Zhan, F.; Huang, Y.; Colla, S.; Stewart, J.P.; Hanamura, I.; Gupta, S.; Epstein, J.; Yaccoby, S.; Sawyer, J.; Burington, B.; et al. The molecular classification of multiple myeloma. Blood 2006, 108, 2020-2028. [CrossRef] [PubMed]

35. Ronchetti, D.; Todoerti, K.; Tuana, G.; Agnelli, L.; Mosca, L.; Lionetti, M.; Fabris, S.; Colapietro, P.; Miozzo, M.; Ferrarini, M.; et al. The expression pattern of small nucleolar and small Cajal body-specific RNAs characterizes distinct molecular subtypes of multiple myeloma. Blood Cancer J. 2012, 2, e96-e103. [CrossRef] [PubMed]

36. Deng, K.; Wang, H.; Guo, X.; Xia, J. The cross talk between long, non-coding RNAs and microRNAs in gastric cancer. Acta Biochim. Biophys. Sin. 2016, 48, 111-116. [CrossRef] [PubMed]

37. Haecker, I.; Gay, L.A.; Yang, Y.; Hu, J.; Morse, A.M.; McIntyre, L.M.; Renne, R. Ago HITS-CLIP expands understanding of Kaposi's sarcoma-associated herpesvirus miRNA function in primary effusion lymphomas. PLoS Pathog. 2012, 8, e1002884-e1002900. [CrossRef] [PubMed]

38. Karginov, F.V.; Hannon, G.J. Remodeling of Ago2-mRNA interactions upon cellular stress reflects miRNA complementarity and correlates with altered translation rates. Genes Dev. 2013, 27, 1624-1632. [CrossRef] [PubMed]

39. Gong, J.N.; Khong, T.; Segal, D.; Yao, Y.; Riffkin, C.D.; Garnier, J.M.; Khaw, S.L.; Lessene, G.; Spencer, A.; Herold, M.J.; et al. Hierarchy for targeting pro-survival BCL2 family proteins in multiple myeloma: Pivotal role of MCL1. Blood 2016, 128, 1834-1844. [CrossRef] [PubMed]

40. Roberts, K.G.; Li, Y.; Payne-Turner, D.; Harvey, R.C.; Yang, Y.L.; Pei, D.; McCastlain, K.; Ding, L.; Lu, C.; Song, G.; et al. Targetable kinase-activating lesions in Ph-like acute lymphoblastic leukemia. N. Engl. J. Med. 2014, 371, 1005-1015. [CrossRef] [PubMed]

41. Simpson, H.M.; Khan, R.Z.; Song, C.; Sharma, D.; Sadashivaiah, K.; Furusawa, A.; Liu, X.; Nagaraj, S.; Sengamalay, N.; Sadzewicz, L.; et al. Concurrent Mutations in ATM and Genes Associated with Common gamma Chain Signaling in Peripheral T Cell Lymphoma. PLoS ONE 2015, 10, e0141906-e0141922. [CrossRef] [PubMed]

42. Xiong, D.; Sheng, Y.; Ding, S.; Chen, J.; Tan, X.; Zeng, T.; Qin, D.; Zhu, L.; Huang, A.; Tang, H. LINC00052 regulates the expression of NTRK3 by miR-128 and miR-485-3p to strengthen HCC cells invasion and migration. Oncotarget 2016, 7, 47593-47608. [CrossRef] [PubMed]

43. Guidi, M.; Muinos-Gimeno, M.; Kagerbauer, B.; Marti, E.; Estivill, X.; Espinosa-Parrilla, Y. Overexpression of miR-128 specifically inhibits the truncated isoform of NTRK3 and upregulates BCL2 in SH-SY5Y neuroblastoma cells. BMC Mol. Biol. 2010, 11, 95-111. [CrossRef] [PubMed]

44. Di Martino, M.T.; Rossi, M.; Caracciolo, D.; Gulla, A.; Tagliaferri, P.; Tassone, P. Mir-221/222 are promising targets for innovative anticancer therapy. Expert. Opin. Ther. Targets 2016, 20, 1099-1108. [CrossRef] [PubMed]

(C) 2016 by the authors; licensee MDPI, Basel, Switzerland. This article is an open access article distributed under the terms and conditions of the Creative Commons Attribution (CC-BY) license (http://creativecommons.org/licenses/by/4.0/). 